\title{
CONSTITUINTES QUÍMICOS DA CASCA DO CAULE DE Amburana cearensis A.C. SMITH
}

Kirley Marques Canuto e Edilberto Rocha Silveira*

Departamento de Química Orgânica e Inorgânica, Centro de Ciências, Universidade Federal do Ceará, CP 12200, 60021-970 Fortaleza - CE, Brasil

Recebido em 26/10/05; aceito em 30/3/06; publicado na web em 22/8/06

\begin{abstract}
CHEMICAL CONSTITUENTS OF TRUNK BARK OF Amburana cearensis A.C. SMITH. The phytochemical analysis of the ethanol extract trunk bark of Amburana cearensis allowed the isolation and identification of twelve constituents: coumarin, sucrose, two phenol acids (vanillic acid and protocatechuic acid), five flavonoids (afrormosin, isokaempferide, kaempferol, quercetin and 4'-methoxy-fisetin), a phenol glucoside (amburoside A) and a mixture of glucosilated $\beta$-sitosterol and stigmasterol. Their structures were elucidated by spectroscopic methods such as IR, MS, ${ }^{1} \mathrm{H}$ and ${ }^{13} \mathrm{C}$ NMR, including uni and bidimensional techniques, in addition to comparison with literature data.
\end{abstract}

Keywords: Amburana cearensis; chemical constitution; spectroscopic data.

\section{INTRODUÇÃO}

Amburana cearensis A.C. Smith (sin. Torresea cearensis Fr. All) pertence à família Leguminoseae Papilionoideae (Fabaceae), sendo ao lado da A. acreana os únicos representantes do gênero. Popularmente, é conhecida por diversas designações, como imburanade-cheiro, cerejeira e cumaru. Esta última denominação, a mais famosa delas, freqüentemente provoca confusões com outra leguminosa homônima chamada Dipteryx odorata, ambas ricas em cumarina (1). Já o termo imburana costuma provocar iguais equívocos na identificação, por se referir também à Commiphora leptophloeos (Burseraceae), conhecida comumente como imburana-de-espinho ${ }^{1}$.

Embora considerada nativa do sertão nordestino, a ocorrência de A. cearensis pode ser observada em praticamente toda América do Sul (do Peru à Argentina) ${ }^{2}$, apresentando-se como uma árvore frondosa, a qual pode atingir até $15 \mathrm{~m}$ de altura, com flores brancas, vagem achatada e casca do caule vermelho-pardacenta cujo agradável odor é conferido pela cumarina. Suas sementes são pretas, aladas e exalam forte cheiro de cumarina (semelhante à baunilha $)^{3}$. No Nordeste, o período de floração ocorre no início da estação seca, entre maio e julho, e a frutificação se dá de agosto a outubro.

Sob o ponto de vista econômico, A. cearensis apresenta inestimável importância comercial dadas suas várias aplicações, sendo largamente empregada na carpintaria e perfumaria. Comercializada com o nome de cerejeira-do-nordeste, sua madeira é utilizada na fabricação de móveis, portas, janelas e caixotaria, devido à reconhecida durabilidade. As sementes servem como aromatizantes e repelentes de insetos para roupas e estantes, podendo também ser utilizadas na fabricação de um pó fino, designado rapé-de-imburana, empregado para induzir espirros no tratamento de "estalecido" (congestão nasal por acúmulo de secreção) ${ }^{1}$.

Na medicina popular, as cascas do caule são tradicionalmente utilizadas na preparação de "lambedôs" caseiros para tratamento de doenças respiratórias, como gripe, resfriado, bronquite e asma ${ }^{4}$. Industrialmente, a forma farmacêutica disponível é o xarope de cumaru, produzido pelo Programa Farmácias Vivas, Farmácia-Escola/UFC e por algumas empresas privadas. Ensaios farmacológicos pré-clínicos demonstraram atividades antiinflamatória, bronco-

*e-mail: edil@ufc.br dilatadora e analgésica para o extrato hidroalcoólico, tendo sido possível ainda atribuir os efeitos observados à cumarina e à fração flavonoídica ${ }^{5,6}$.

Em virtude do uso difundido de A. cearensis para fins medicinais e da escassez de relatos fitoquímicos na literatura, tornou-se imprescindível a realização do seu estudo, visando a descoberta dos possíveis responsáveis pelas propriedades terapêuticas da espécie. Este trabalho relata a metodologia de isolamento e a identificação de 12 constituintes químicos presentes no extrato etanólico das cascas do caule de A. cearensis.

\section{RESULTADOS E DISCUSSÃO}

Levantamento bibliográfico revelou que, apesar da ação comprovada e do uso difundido do xarope de cumaru, somente 4 constituintes químicos [cumarina (1), isocampferídio (5), amburosídio A (10) e amburosídio B (11)] haviam sido relatados na literatura para as cascas do caule de $A$. cearensis $^{4,7}$.

Sucessivos tratamentos cromatográficos em gel de sílica, e/ou sobre dextrana da fração acetato de etila, obtida por partição $\mathrm{H}_{2} \mathrm{O}$ / solvente orgânico (Hexano, AcOEt) do extrato etanólico das cascas do caule de A. cearensis, resultaram no isolamento e na identificação de 11 constituintes químicos: cumarina, dois ácidos fenólicos [ácido vanílico (4) e ácido protocatecuico (6)], um heterosídio fenólico (amburosídio A), a mistura de $\beta$-sitosterol e estigmasterol glicosilados (8 e 9) e cinco flavonóides [afrormosina (3), isocampferídio, campferol (7), quercetina (12), 4'-metoxi-fisetina (13)], dos quais quatro são flavonóis, sendo o isocampferídio um derivado do campferol metilado na posição 3, isômero da 4'-metoxifisetina. Além disso, a partir do extrato etanólico ainda se obteve sacarose (2) por precipitação, após resfriamento da solução etanólica.

As substâncias isoladas tiveram suas estruturas elucidadas através de métodos espectroscópicos, tais como IV, EM e RMN ${ }^{1} \mathrm{H}$ e ${ }^{13} \mathrm{C}$, incluindo técnicas uni e bidimensionais (COSY, HMQC e HMBC), e corroboradas por comparação com dados descritos na literatura. A pureza foi constatada por cromatoplacas analíticas e HPLC.

Algumas das frações e substâncias obtidas no presente estudo foram submetidas a diferentes testes farmacológicos, cujos resultados foram publicados previamente. A fração flavonoídica, tendo o isocampferídio como constituinte majoritário, e a cumarina demons- 
traram atividade antiinflamatória mais potente que a do extrato hidroalcoólico, ao produzirem maior inibição na permeabilidade vascular e na migração de neutrófilos e leucócitos ${ }^{6}$. O isocampferídio e o campferol exibiram significativa atividade citotóxica contra ovos de ouriço-do-mar e cinco linhagens de células tumorais (dois tipos de células leucêmicas humanas, células cancerosas de mama, cólon e pele) em ensaios in vitro ${ }^{8}$. $\mathrm{O}$ amburosídio demonstrou propriedades neuroprotetoras em células mesencefálicas, agindo como antioxidante contra a neurotoxina 6-hidroxi-dopamina9 ${ }^{9}$<smiles>O=c1ccc2ccccc2o1</smiles>

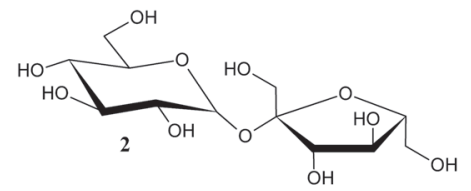

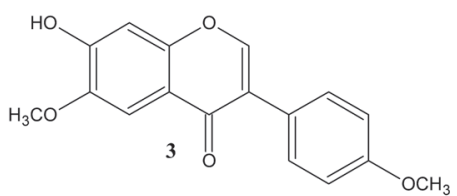<smiles>[R20]c1ccc(-c2oc3cc(O)cc([R2])c3c(=O)c2OCC)cc1[R6]</smiles>

$$
\begin{array}{lllll} 
& \mathrm{R}_{1}= & \mathrm{R}_{2}= & \mathrm{R}_{3}= & \mathrm{R}_{4}= \\
\mathbf{5} & \mathrm{CH}_{3} & \mathrm{OH} & \mathrm{H} & \mathrm{H} \\
\mathbf{7} & \mathrm{H} & \mathrm{OH} & \mathrm{H} & \mathrm{H} \\
\mathbf{1 2} & \mathrm{H} & \mathrm{OH} & \mathrm{OH} & \mathrm{H} \\
\mathbf{1 3} & \mathrm{H} & \mathrm{H} & \mathrm{OH} & \mathrm{CH}_{3}
\end{array}
$$

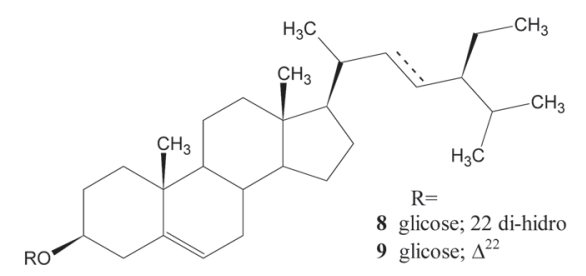<smiles>[R6]c1cc(C(=O)OCc2ccc(OC3O[C@H](CO)[C@@H](O)[C@H](O)[C@H]3O)cc2)ccc1O</smiles>

Figura 1. Estruturas químicas das substâncias isoladas de Amburana cearensis

\section{CONCLUSÃO}

O estudo da constituição química da casca do caule de $A$. cearensis revelou a presença profusa de compostos fenólicos na planta, principalmente flavonóides, podendo estes serem apontados, ao lado da cumarina, como responsáveis pelas atividades farmacológicas da espécie, conforme efeitos observados em testes realizados com as substâncias puras ${ }^{6,8,9}$. O aprofundamento no conhecimento químico e farmacológico desta planta deve ser considerado de incontestável importância sócio-econômica, pois certamente permitirá, em futuro próximo, a formulação de produtos mais eficazes e seguros para a população, tanto para os adeptos da fitoterapia como para empresários do ramo.

\section{PARTE EXPERIMENTAL}

\section{Procedimentos experimentais gerais}

Os espectros de RMN ${ }^{1} \mathrm{H}$ e ${ }^{13} \mathrm{C}$, uni e bidimensionais, foram obtidos em espectrômetros Bruker, modelos Avance DPX-300 e/ou Avance DRX-500 (CENAUREMN-UFC), operando na freqüência do hidrogênio a 300,13 e a $500,13 \mathrm{MHz}$, e na freqüência do carbono a 75,47 e 125,75 MHz, respectivamente. Solventes deuterados foram empregados para dissolução das amostras. Os espectros de absorção na região do IV foram obtidos em espectrômetro PerkinElmer, modelo FT-IR Spectrum 1000, utilizando pastilhas de $\mathrm{KBr}$ para análise das amostras. Os espectros de massa foram registrados em espectrômetros Shimadzu, modelo QP 5000/DI-50, através de impacto eletrônico a 70 eV e Micromass Quattro-LC-QC9425, através de ionização por "eletrospray”. Os pontos de fusão, não corrigidos, foram determinados em equipamento da Mettler Toledo, composto por uma placa aquecedora FP82HT e uma unidade de processamento FP90, a uma taxa de aquecimento de $2{ }^{\circ} \mathrm{C} / \mathrm{min}$. As rotações ópticas foram determinadas em polarímetro Perkin-Elmer, modelo 341 , sendo as medidas realizadas a $589 \eta \mathrm{m}\left(20^{\circ} \mathrm{C}\right)$.

As cromatografias de adsorção foram realizadas em coluna sobre gel de sílica 60 da Vetec $(\varnothing \mu \mathrm{m}$ 63-200). Os fracionamentos por cromatografia de exclusão foram efetuados em gel de dextrana Sephadex LH-20 da Pharmacia Fine Chemicals. As separações foram monitoradas por cromatoplacas de gel de sílica 60 ( $\varnothing \mu \mathrm{m} 2-25)$ Sigma Chemical CO (com indicador de fluorescência na faixa de $254 \mathrm{\eta m}$ ) e em um aparelho de HPLC Waters-1525 acoplado a um detector de PDA Waters-2996 (254 nm), utilizando coluna XTerra RP-18 (4,6 x 250 mm, $5 \mu \mathrm{M})$. As substâncias puras foram eluídas com solução aquosa de $\mathrm{H}_{3} \mathrm{PO}_{4}-\mathrm{Et}_{3} \mathrm{~N}(\mathrm{pH}=3,0)$ mais $\mathrm{MeOH}$, variando de 20 a $100 \%$ em 40 min de corrida, adotando um fluxo de $1 \mathrm{~mL} / \mathrm{min}$.

A revelação das substâncias foi produzida por exposição à luz UV (254 e 366 ๆm) em aparelho Spectroline, modelo ENF-240 C/F, e por aspersão com solução ácida de vanilina, seguida de aquecimento.

\section{Material vegetal}

As cascas do caule de A. cearensis foram coletadas na Fazenda São Vicente, no município de Quixeramobim-CE em março de 2000 e setembro de 2002, pela Prof ${ }^{a}$. L. K. A. Leal, pertencente ao Departamento de Farmácia/UFC. A identificação botânica foi fornecida pelo Prof. A. G. Fernandes, do Departamento de Biologia/UFC, encontrando-se sua respectiva exsicata ${ }^{\circ} 847$ depositada no Herbário Prisco Bezerra/UFC.

\section{Extração e isolamento}

3,4 kg de cascas do caule de A. cearensis, secas e moídas, foram divididas em alíquotas de 200 g e submetidas à extração com etanol em aparelho Sohxlet. A solução etanólica, ao ser resfriada, apresentou a formação de cristais marrons $(500 \mathrm{mg})$, os quais foram separados por filtração e analisados por métodos espectroscópicos, por meio dos quais foram identificados como sacarose [2; p.f. 174,6175,5 ${ }^{\circ} \mathrm{C}$ (lit.:160-186 ${ }^{\circ} \mathrm{C}$ ) $]^{10,11}$. Após completa evaporação do solvente, obteve-se um sólido viscoso escuro denominado ACE (205,4 g). ACE foi solubilizado em água $(150 \mathrm{~mL})$ e particionado com AcOEt (120 mL, 4x), gerando 74,2 g da fração AcOEt (ACEA) e 127, $8 \mathrm{~g}$ da fração aquosa, após evaporação/liofilização dos respectivos solventes. ACEA foi dissolvida em $\mathrm{MeOH}(180 \mathrm{~mL})$ e extraída com hexano $(120 \mathrm{~mL}, 4 \mathrm{x})$, fornecendo as frações metanólica (ACEAM- 68,3 g) e hexânica (ACEAH- 6,2 g), respectivamente. 
Na fração ACEAH houve a precipitação de um material que, depois de filtrado e lavado com Hexano/ $\mathrm{CHCl}_{3}(1: 1)$, rendeu o sólido incolor 1, caracterizado como cumarina [790 mg; p.f. 67,8-68,7 ${ }^{\circ} \mathrm{C}$ (lit.: $68-70{ }^{\circ} \mathrm{C}$ )] cujo tempo de retenção (TR) em análise por HPLC foi de 13,7 min $)^{11,12}$. A fração ACEAM foi adsorvida em gel de sílica (75 g) e eluída com: $\mathrm{CHCl}_{3}(1,0 \mathrm{~L}) ; \mathrm{CHCl}_{3} / \mathrm{AcOEt} 1: 1(1,0$ L); AcOEt (1,7 L) e MeOH (0,5 L), resultando em ACEAM/C (7,5 g); ACEAM/CA (19,1 g); ACEAM/A(18,3 g) e ACEAM/M (23,5 g), respectivamente.

A fração ACEAM/CA foi cromatografada em gel de sílica (114 g), utilizando gradiente de eluição composto por $\mathrm{CHCl}_{3} / \mathrm{AcOEt}$. Foram coletadas 23 alíquotas de $75 \mathrm{~mL}$ e reunidas as frações semelhantes, identificadas por CCD. A cromatografia em Sephadex LH$20(60 \mathrm{~g})$ da fração $8-9(452 \mathrm{mg})$, obtida por eluição com $\mathrm{CHCl}_{3} /$ AcOEt (8:2), foi efetuada com metanol, coletando-se 15 frações de $8 \mathrm{~mL}$. Destas, somente a fração 7 conduziu ao isolamento de 3 [afrormosina: sólido amorfo branco, 22,6 mg; p.f. $230,5-232,0{ }^{\circ} \mathrm{C}$ (lit. $\left.228-229{ }^{\circ} \mathrm{C}\right)^{13-15}$. A fração $10-14(1,64 \mathrm{~g})$, obtida por eluição com $\mathrm{CHCl}_{3} / \mathrm{AcOEt}(8: 2)$ e $(7: 3)$, foi submetida à cromatografia em Sephadex LH-20 (60 g) com MeOH. Foram coletadas 30 frações de $8 \mathrm{~mL}$, das quais as frações 15 e 16 resultaram no isolamento de 4 [ácido vanílico: cristais amarelados, 46,1 mg; TR = 10,3 min; p.f. 204,9-205,8 ${ }^{\circ} \mathrm{C}$ (lit.: $210{ }^{\circ} \mathrm{C}$ ) $]^{11,12}$, enquanto que as frações 9-13 levaram à obtenção de $\mathbf{5}$ [isocampferídio: sólido amorfo amarelo, 143 mg; TR = 28,7 min; p.f. $290,7-292,1{ }^{\circ} \mathrm{C}$ (lit.: 299-302 ${ }^{\circ} \mathrm{C}$; 250-253 $\left.\left.{ }^{\circ} \mathrm{C}\right)\right]^{13,16,17}$. A subfração (10-14)(18) (350 mg), proveniente desta última cromatografia, foi recristalizada em $\mathrm{CHCl}_{3} / \mathrm{AcOEt}(1: 1)$, obtendo-se 6 [ácido protocatecuico, $229 \mathrm{mg}$; TR = 7,0 min; p.f. 199,2200, $7^{\circ} \mathrm{C}$ (lit.: $200^{\circ} \mathrm{C}$ ) na forma de cristais aciculares ${ }^{11,12}$. A subfração (10-14)(19-24) (205 mg) foi recromatografada em Sephadex LH-20 com metanol, sendo coletadas 15 frações de $5 \mathrm{~mL}$. As frações 9 e 10 foram identificadas como 7 [campferol: sólido amorfo amarelo; 44,3 mg; TR = 29,2 min; p.f. $275,5-277,1^{\circ} \mathrm{C}$ (lit. 276-278 ${ }^{\circ} \mathrm{C}$ ) $]^{13,14}$. Uma alíquota de $4 \mathrm{~g}$ de ACEAM/A foi cromatografada em Sephadex LH20, sendo obtidas 20 frações de $10 \mathrm{~mL}$ de $\mathrm{MeOH}$. A fração 4-5, um sólido amorfo branco $(7 \mathrm{mg})$, foi identificada como uma mistura de 8 e 9 ( $\beta$-sitosterol e estigmasterol glicosilados) ${ }^{18}$. Após sucessivos fracionamentos em Sephadex LH-20, a fração 6-8, um sólido escuro, foi lavado com AcOEt/MeOH (1:1) e filtrado, obtendo-se ao final um sólido rosado caracterizado como $\mathbf{1 0}$ [amburosídio A, 250 mg; TR = 14,5 min; p.f. $197,8-199,1{ }^{\circ} \mathrm{C}$ (lit.: $\left.195-197{ }^{\circ} \mathrm{C}\right)^{7}$.

Repetindo-se a metodologia de partição do extrato etanólico ACE $(192,4 \mathrm{~g})$, obtido de cascas do caule $(4,0 \mathrm{~kg})$ em uma nova coleta (setembro/2002), e subseqüiente cromatografia em gel de sílica da fração ACEAM $(64,5 \mathrm{~g})$, gerou-se a fração $\operatorname{ACEAM/C~}(2,1 \mathrm{~g})$ que, ao ser recristalizada em hexano/ $\mathrm{CHCl}_{3} 1: 1$, resultou novamente no isolamento de $\mathbf{1}(822,7 \mathrm{mg})$. A fração ACEAM/CA (22,7 g) foi submetida à cromatografia em gel de sílica (160 g), empregando misturas de $\mathrm{CHCl}_{3} / \mathrm{AcOEt}$ com polaridade ascendente. Foram coletadas 33 frações de $75 \mathrm{~mL}$, sendo as frações 24-31 (4,0 g), eluídas com $\mathrm{CHCl}_{3} / \mathrm{AcOEt}(6: 4)$ e (1:1), reunidas após serem comparadas por CCD. Cromatografia de f24-31 em Sephadex LH-20 (60 g), por eluição com $\mathrm{MeOH}$, forneceu 35 frações de $10 \mathrm{~mL}$, sendo a penúltima delas um sólido amarelo identificado como $\mathbf{1 2}$ [quercetina, 41,2 mg; TR = 25,7 min.; p.f. $>300^{\circ} \mathrm{C}$ (lit.: $314^{\circ} \mathrm{C}$ ) $]^{11,19}$. As frações iniciais (2-8) (2,4 g), resultantes desta cromatografia, foram reunidas mediante comparação por cromatoplaca, e novamente cromatografadas em Sephadex LH-20 (60 g). Coletaram-se 20 frações de $10 \mathrm{~mL}$, usando $\mathrm{MeOH}$ como eluente. As frações 4-6 (186 $\mathrm{mg})$ e 8-13 (954 mg) foram reconhecidas como as substâncias já isoladas 6 e 10, respectivamente, através de análise comparativa por CCD com padrões autênticos.

Nova extração das cascas do caule $(2,2 \mathrm{~kg})$, remanescentes da coleta anterior, rendeu $122 \mathrm{~g}$ do extrato etanólico ACE, que submetido à mesma metodologia de partição e cromatografia de adsorção, resultou na fração ACEAM/CA(4-9) (155,6 mg), obtida por eluição com $\mathrm{CHCl}_{3} / \mathrm{AcOEt}$ (9:1) e (8:2). Fracionamento em Sephadex LH-20 (30 g) de ACEAM/CA(4-9), utilizando $\mathrm{MeOH}$ como eluente, originou 15 frações de $5 \mathrm{~mL}$. Na fração 9, obteve-se um sólido amarelo que, ao ser analisado por CCD, apresentou uma intensa fluorescência sob UV, sendo caracterizado como 13 [4'-metoxi-fisetina, $5,8 \mathrm{mg}$; p.f. $>300{ }^{\circ} \mathrm{C}$ (lit. $288^{\circ} \mathrm{C}$ ) $]^{13,14}$, por técnicas espectrométricas.

\section{AGRADECIMENTOS}

Ao $\mathrm{CNPq}$ pelas bolsas concedidas, bem como à CAPES/CNPq/ FUNCAP/PADCT/FINEP/BNB pelo suporte financeiro.

\section{REFERÊNCIAS}

1. Maia, G. N.; Caatinga: Árvores e Arbustos e Suas Utilidades, D \& Z Ed.: São Paulo, 2004.

2. Carvalho, P. E. R.; Espécies Florestais Brasileiras: Recomendações Silviculturais, Potencialidades e Uso da Madeira, EMBRAPA: Brasília, 1994.

3. Pio-Corrêa, M.; Dicionário de Plantas Úteis do Brasil e das Exóticas Cultivadas, Ministério da Agricultura: Brasília, 1984.

4. Leal, L. K. A. M.; Dissertação de Mestrado, Universidade Federal do Ceará, Brasil, 1995.

5. Leal, K. A. M.; Matos, M. E.; Matos, F. J. A.; Ribeiro, R. A.; Ferreira, F. V.; Viana, G. S. B.; Phytomedicine 1997, 4, 221.

6. Leal, L. K. A. M.; Nechio, M.; Silveira, E. R.; Canuto, K. M.; Fontenele, J. B.; Ribeiro, R. A.; Viana, G. S. B.; Phytother. Res. 2003, 17, 335.

7. Bravo, J. A. B.; Sauvain, M.; Gimenez, A.; Muñoz, V.; Callapa, J.; Le MenOlivier, L.; Massiot, G.; Lavaud, C.; Phytochemistry 1999, 50, 71.

8. Costa-Lotufo, L. V.; Jimenez, P. C.; Wilke, D. V.; Leal, L. K. A. M.; Cunha, G. M. A.; Silveira, E. R.; Canuto, K. M.; Viana, G. S. B.; Moraes, M. E. A.; Moraes, M. O.; Pessoa, C.; Z. Naturforsch., C: J. Biosci. 2003, 58, 675.

9. Leal, L. K. A. M.; Nobre-Júnior, H. V.; Cunha, G. M. A.; Moraes, M. O., Pessoa, C.; Oliveira, R. A.; Silveira, E. R.; Canuto, K. M.; Viana, G. S. B.; Neurosci. Lett. 2005, 388, 86.

10. Breitmaier, E; Voelter, W.; ${ }^{13}$ C NMR Spectroscopy: Methods and Applications, Verlag Chemie: Düsseldorf, 1974.

11. Budavari, S.; O`Neil, M. J.; Smith, A.; Heckelman, P. E.; Kinneary, J. F.; The Merck Index- An Encyclopedia of Chemical, Drugs and Biologicals, $12^{\text {th }}$ ed., Merck Co: New Jersey, 1996.

12. Pouchert, C. J.; Behnke, J.; The Aldrich Library of ${ }^{13} \mathrm{C},{ }^{1} \mathrm{H}$ FT NMR Spectra, $1^{\text {st }}$ ed., Aldrich Chemical Company: Milwaukee, 1993.

13. Agrawal, P. K.; Carbon-13 NMR of Flavonoids, Elsevier: New York, 1989.

14. Djerassi, C.; Connoly, J. D.; Faulkner, D. J.; Mori, K.; Nakanishi, K.; Ourisson, G.; Raphael, R. A.; Shamma, M.; Tamm, C. H.; Dictionary of Natural Products, $1^{\text {st }}$ ed., Chapman \& Hall: London, 1994.

15. Herath, H. M. T. B.; Dassanayake; R. S.; Priyadarshani, A. M. A.; Wannigama, G. P.; Phytochemistry 1998, 47, 117.

16. Sim, K. Y.; Phytochemistry 1969, 8, 1597.

17. Ganzera, M.; Elmerer-Müller, E. P.; Stuppner, H.; Phytochemistry 1998, 49, 835 .

18. Kojima, H.; Sato, N.; Hatano, A.; Ogura, H.; Phytochemistry 1990, 29 , 2351.

19. Oliveira, M. C.; Carvalho, M. G.; Ferreira, D. T.; Braz-Filho, R.; Quim. Nova 1999, 22, 182. 